\title{
ARTICLE
}

\section{Hyperpolarized nanodiamond with long spin-relaxation times}

\author{
Ewa Rej ${ }^{1, \star}$, Torsten Gaebel ${ }^{1, \star}$, Thomas Boele ${ }^{1}$, David E.J. Waddington ${ }^{1} \&$ David J. Reilly ${ }^{1}$
}

The use of hyperpolarized agents in magnetic resonance, such as ${ }^{13} \mathrm{C}$-labelled compounds, enables powerful new imaging and detection modalities that stem from a 10,000-fold boost in signal. A major challenge for the future of the hyperpolarization technique is the inherently short spin-relaxation times, typically $<60 \mathrm{~s}$ for ${ }^{13} \mathrm{C}$ liquid-state compounds, which limit the time that the signal remains boosted. Here we demonstrate that $1.1 \%$ natural abundance ${ }^{13} \mathrm{C}$ spins in synthetic nanodiamond can be hyperpolarized at cryogenic and room temperature without the use of free radicals, and, owing to their solid-state environment, exhibit relaxation times exceeding $1 \mathrm{~h}$. Combined with the already established applications of nanodiamonds in the life sciences as inexpensive fluorescent markers and non-cytotoxic substrates for gene and drug delivery, these results extend the theranostic capabilities of nanoscale diamonds into the domain of hyperpolarized magnetic resonance.

\footnotetext{
${ }^{1}$ ARC Centre of Excellence for Engineered Quantum Systems, School of Physics, University of Sydney, Sydney, New South Wales 2006, Australia. * These authors contributed equally to this work. Correspondence and requests for materials should be addressed to D.J.R. (email: david.reilly@sydney.edu.au).
} 
$\mathrm{N}$ anoparticles, having found use in the treatment of cancers $^{1,2}$, the study of autoimmune diseases ${ }^{3}$ and cardiovascular affections ${ }^{4}$, are currently of interest as theranostic agents needed for the advent of personalized medicine ${ }^{5}$. These nanoscale systems are expected to integrate imaging, tracking and monitoring capabilities with targeted delivery of compounds to tumours, cellular functions and processes, or specific organs. Especially powerful is the modality established by combining high-resolution magnetic resonance imaging (MRI) with nanoparticles that have been hyperpolarized to act as contrast agents, as has been achieved recently using silicon compounds 6,7 .

Nanodiamonds (NDs) are well suited to act as theranostic platforms, having demonstrated an innate compatibility with biological environments and low toxicity in comparison with other nanoscale structures ${ }^{8,9}$. The readily modifiable surface, which is easily functionalized ${ }^{10}$, has enabled NDs to be conjugated to specific molecules ${ }^{11}$, opening a plethora of biomedical applications that include pharmaceutical delivery $9,12-14$ and intracellular tracking ${ }^{15}$ based on the unique optical properties of defects in the diamond lattice ${ }^{11}$. A particular defect, the nitrogen vacancy colour centre, has also established a sensitive means of detecting minute magnetic fields on the nanoscale using methods pioneered in controlling quantum devices $^{16-22}$. Beyond luminescence-based techniques, however, approaches to non-invasively detect and image diamond nanoparticles in vivo have to date, been lacking.

Standard MRI modalities (operating at few Tesla magnetic fields) are not well suited for resolving weak concentrations of $\mathrm{ND}$ in vivo since diamond is a dilute spin system $\left(1.1 \%{ }^{13} \mathrm{C}\right)$ and carbon has a small gyromagnetic ratio. This limitation can, in principle, be overcome using hyperpolarization techniques ${ }^{23}$, which can result in a 10,000 -fold boost in signal over that from typical thermal polarization conditions ${ }^{24,25}$. Hyperpolarized molecular compounds such as $\left[1-{ }^{13} \mathrm{C}\right]$ pyruvate, for example, have recently been used to study tumour metabolism in humans by first transferring electron spin polarization to ${ }^{13} \mathrm{C}$ nuclei at cryogenic temperatures ${ }^{26}$.

In these liquid-state compounds, hyperpolarized ${ }^{13} \mathrm{C}$ spins typically relax to thermal equilibrium on timescales $T_{1}<60 \mathrm{~s}$ (ref. 23). In contrast, bulk, high-purity diamond can exhibit ${ }^{13} \mathrm{C}$ $T_{1}$ times of many hours ${ }^{27}$ and recent work using optical techniques to manipulate nitrogen vacancy centres $28-31$ has produced significant polarization in large single-crystal samples. The challenge therefore is to maintain these long spin lifetimes even when diamond is produced in nanoparticle form and in sufficient quantities to be of clinical relevance. Addressing this challenge requires a detailed understanding of particle size effects, the structure of internal crystal defects, contaminants and spin-relaxation channels that arise from the nanoparticle surface $^{32-35}$. Balancing these constraints, the hyperpolarization mechanism also requires the presence of unpaired electrons which in the case of liquid ${ }^{13} \mathrm{C}$ compounds, are typically added to the agent in the form of organic free radicals.

In the present work, we extend the opportunity for deploying ND in life-science applications by demonstrating its suitability as a magnetic resonance marker and contrast agent for MRI. Using electron spin resonance (ESR), we observe that inexpensive commercially available ND, produced via the high-pressure high-temperature process, surprisingly exhibits a suitable balance of paramagnetic centres from defects and surface dangling bonds to allow both hyperpolarization and the preservation of long spin-relaxation times. In comparison with previous results on detonation $\mathrm{ND}^{32,33}$, the ${ }^{13} \mathrm{C}$ relaxation data reported here exhibit a 1,000-fold extension in $T_{1}$ together with signal enhancements that compare favourably with hyperpolarized ${ }^{13} \mathrm{C}$ liquid-state compounds. Particle size is found to significantly affect both the relaxation time and amount of achievable hyperpolarization, opening the possibility of selectively detecting NDs of a particular size distribution. In addition to showing significant hyperpolarization at $T=4 \mathrm{~K}$, we demonstrate that a sizeable signal enhancement is also possible at liquid nitrogen $(77 \mathrm{~K})$ and room temperature using dynamic nuclear polarization (DNP), alleviating the need for expensive liquid helium and potentially enabling new in vivo modalities. Finally, we examine the spin dynamics of the ND core and its surface using hyperpolarized states to resolve new phenomena associated with defects in this versatile material system.

\section{Results}

ESR spectra and nuclear spin relaxation. Turning to the experimental results, scanning electron microscopy (SEM) images (Fig. 1a-d; Supplementary Fig. 1) and the ESR spectrum and simulation results (Fig. 1e) for a representative ND sample are shown. The ESR spectrum, which indicates the predominant types of defects available for use in hyperpolarization, can be seen to comprise three components that sum to produce the black simulation curve in Fig. 1e. These are a broad spin-1/2 Lorentzian component (blue trace) attributed to carbon dangling bonds near the surface of the ND, a narrow spin-1/2 Lorentzian component (yellow trace) attributed to defects within the diamond lattice, and a component associated with P1-colour centres (green trace), which constitutes a substitutional nitrogen atom with the extra electron hyperfine coupled with the ${ }^{14} \mathrm{~N}$ spin-1 nucleus. The number of P1-centre impurities, which lead to central $\left(m_{\mathrm{I}}=0\right)$ and hyperfine transitions $\left(m_{\mathrm{I}}= \pm 1\right)$, increases as ND size increases, while the number of spin-1/2 impurities (broad and narrow spectra) decreases as ND size increases (Supplementary Figs 2 and 3).

These defect sites also provide the primary mechanism for ${ }^{13} \mathrm{C}$ nuclear spin relaxation in ND. We find that the $T_{1}$ relaxation time grows with increasing particle size, as shown in Fig. 1f. In determining these $T_{1}$ times, the spin polarization build-up for smaller diamonds is well described by models ${ }^{36,37}$ in which the dipolar interaction of nuclear spins with paramagnetic impurities dominates over nuclear spin diffusion, leading to polarization curves that follow a stretched exponential form (see the Methods section). Diamond particles with average diameter approaching $1 \mu \mathrm{m}$, however, are better characterized by a double exponential in their polarization build-up with time. For $2 \mu \mathrm{m}$ diamonds the longer component of the double exponential yields a $T_{1}$ time of $63 \mathrm{~min}$ (see the Methods section and Supplementary Figs 4 and 5).

Brute force hyperpolarization. The simplest method of increasing the magnetic resonance signal from ND is to first cool the system to low temperatures in a high magnetic field to increase the Boltzmann population difference in the nuclear spins, a process termed brute force polarization. If the NDs are subsequently moved to a different magnetic field and temperature, the spin system can be considered hyperpolarized until it thermalizes on timescale $T_{1}$. Using the brute force method, we hyperpolarize $2-\mu \mathrm{m} \mathrm{ND}$ at $T=35 \mathrm{mK}$ and $B=4 \mathrm{~T}$ in a dilution refrigerator fitted with a rapid sample exchange system that allows fast $(<1 \mathrm{~min})$ transfer of the $\mathrm{ND}$ sample to a room temperature $B=7 \mathrm{~T}$ spectrometer for detection. A $\pi / 2$-pulse applied immediately after transfer produces a signal (Fig. 1g, inset) that is enhanced by an order of magnitude when compared with the signal from $2-\mu \mathrm{m} N$ at thermal equilibrium and $B=7 \mathrm{~T}$. To measure the relaxation time, a series of small tip angles is used to destroy the polarization over $0.5 \mathrm{~h}$, as indicated 
a

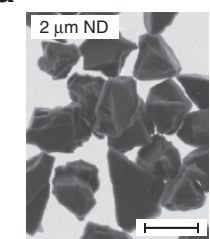

C
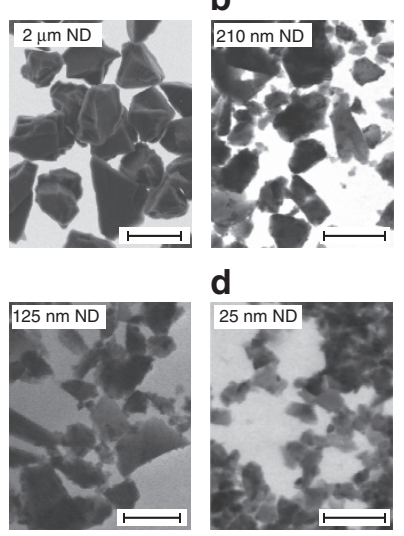

e

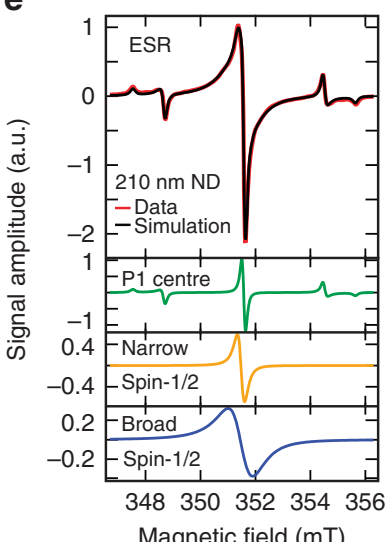

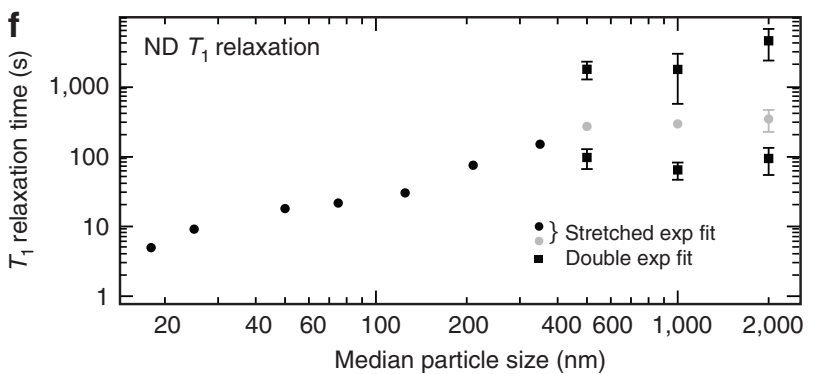

g

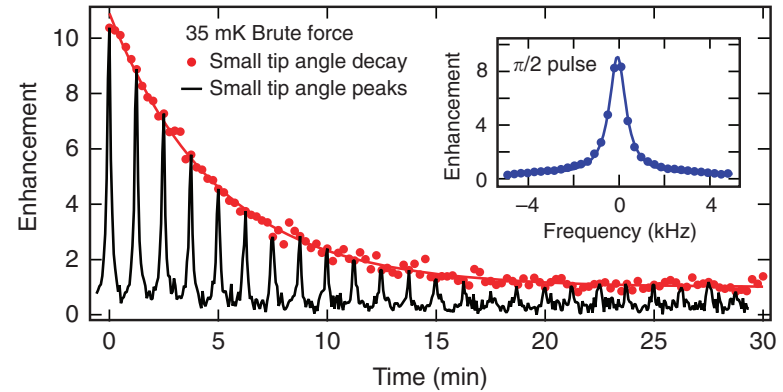

Figure 1 | ND characterization and brute force hyperpolarization.

(a-d) Electron micrographs of various sized NDs used in this work. (a) $2 \mu \mathrm{m}$

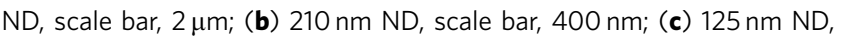
scale bar, $200 \mathrm{~nm}$; (d) $25 \mathrm{~nm}$ ND, scale bar, $100 \mathrm{~nm}$. (e) ESR spectrum of $210 \mathrm{~nm}$ ND (red). The black line is a simulated spectrum consisting of three components: a narrow spin-1/2 component (yellow), a broad spin-1/2 component (blue) and a P1-centre component (green). (f) Room temperature ${ }^{13} \mathrm{C}$ relaxation times, $T_{1}$, as a function of diamond particle size at $B=7 \mathrm{~T}$. Relaxation times were measured using the saturation recovery method with build-up time of magnetization $M$ determined by fitting a stretched exponential $M=M_{0}\left(1-\exp \left(-\left(t / T_{1}\right)^{\alpha}\right)\right.$ or double exponential. Small NDs exhibit a build-up with $\alpha=2 / 3$ (black circles), with larger NDs better fitted by a stretched exponential with $\alpha=1 / 2$ (grey circles) or double exponential fits with a long and short component shown as black squares. The error bars indicate the s.d. of the three measurements that were taken, and are shown when the error exceeds the size of the marker. $(\mathbf{g})$ Enhanced signal following brute force hyperpolarization of $2-\mu \mathrm{m} \mathrm{ND}$ at $T=35 \mathrm{mK}$ and $B=4 \mathrm{~T}$ for 3 days in a dilution refrigerator. Following a $40 \mathrm{~s}$ transfer in a field of $630 \mathrm{mT}$, detection is at $B=7 \mathrm{~T}$, via a $\pi / 2$-pulse (inset) with decay ( $T_{1} \sim 53 \mathrm{~min}$ ) measured via a sequence of small tip angles (main panel).

by the decaying signal in Fig. 1g. The decay is a combination of $T_{1}$ relaxation of spins in the $\mathrm{ND}(\sim 53 \mathrm{~min})$ and polarization lost from the tipping pulses (see the Methods section for details).

Hyperpolarization via the solid effect. To achieve even higher polarizations and larger signals, $\mathrm{DNP}^{38}$ can be used to transfer

electron polarization to the ${ }^{13} \mathrm{C}$ nuclear spins in the diamond ${ }^{27}$. As described above, the source of these unpaired electrons in $\mathrm{ND}$ are paramagnetic centres in the lattice, dipolar coupled with a surrounding nuclear spin bath. Application of a microwave magnetic field slightly below the ESR frequency can drive spin flip-flops between nuclear and electron spins associated with centres, leading to a net transfer of spin polarization from the electrons to the nuclei near the impurities in a process known as the solid effect (Fig. 2a,b).

Turning to the main results of our work, we demonstrate that DNP can be used to hyperpolarize commercially available NDs, which as we have shown above, also exhibit long relaxation times. In the case of the largest diameter diamonds $(2 \mu \mathrm{m})$ a $T=4 \mathrm{~K}$ signal enhancement of $\sim 400$ is achieved over thermal equilibrium, corresponding to a nuclear polarization of $\sim 8 \%$, as shown in Fig. 2c-e. Comparing this hyperpolarized signal with the thermal signal at room temperature gives an enhancement of 13,500 similar to what has been demonstrated with isotopically labelled ${ }^{13} \mathrm{C}$ liquid compounds ${ }^{23}$. We estimate that for a ND concentration of $1 \mathrm{mg} \mathrm{ml}^{-1}$, this amount of hyperpolarization leads to a spatial resolution better than $2 \times 2 \mathrm{~mm}$ in a preclinical MRI scanner (Supplementary Note 1). Note that ND exceeding this concentration has already been used for therapeutic delivery in vivo ${ }^{12}$.

It is possible that by hyperpolarizing the nuclear spin system using DNP, new relaxation channels are created that shorten the relaxation time. We test this possibility by first polarizing $2-\mu \mathrm{m}$ $\mathrm{ND}$ for $1 \mathrm{~h}$ and then allowing it to decay for $1 \mathrm{~h}$ at field. The resulting signal, shown in Fig. $2 \mathrm{f}$, indicates a $T_{1}$ comparable to measurements performed at thermal equilibrium. As a further demonstration of the potential for hyperpolarized ND, we show in Fig. $2 \mathrm{~g}$ that the enhanced polarization can be maintained during transfer of the sample from a lower field polarizer to a high-field magnetic resonance detection system (see the Methods section for details).

Unlike hyperpolarized molecular compounds, the use of nanoparticles opens a new modality that links magnetic resonance signal strength (and relaxation time) to particle size. For hyperpolarized ND, we determine a significant size dependence to the signal enhancement, as shown in Fig. 2h. This dependence is most prominent for particle sizes below $\sim 300 \mathrm{~nm}$, where the larger rate of spin relaxation competes with the rate at which hyperpolarization from DNP occurs. We suggest that this dependence on the diameter of NDs opens a means of selectively tracking particles based on their size distribution, potentially of use in determining the integrity of permeable barriers and epithelium membranes.

For potential clinical use of hyperpolarized MRI, a major drawback of the technique is the need for liquid helium to cool sample agents during the polarization phase. This drawback is particularly significant for applications that require magnetic resonance in remote locations, for instance in battlefield MRI using ultralow magnetic fields. In the case of hyperpolarized ND however, we find that sizeable enhancements are possible at liquid nitrogen temperatures (77 K; Fig. 2h), where cryogens are readily available. Extending this idea, Fig. $2 \mathrm{~h}$ also shows that hyperpolarization is possible at room temperature, doing away with cryogens altogether.

Hyperpolarization in the presence of water. Room temperature hyperpolarization, even for modest enhancements, opens the prospect of new modalities that polarize and detect compounds in vivo. The significant barrier to this technique is the heating of water and surrounding tissue during the application of microwaves needed to perform DNP. Polarizing at low magnetic field and microwave frequency, however, can significantly reduce 
a
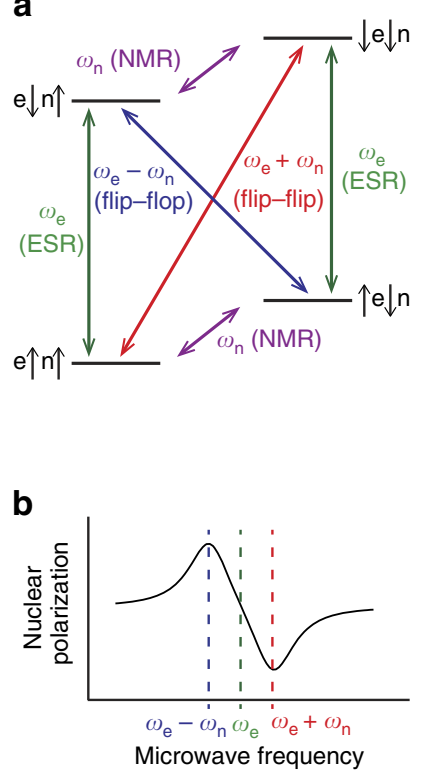

C
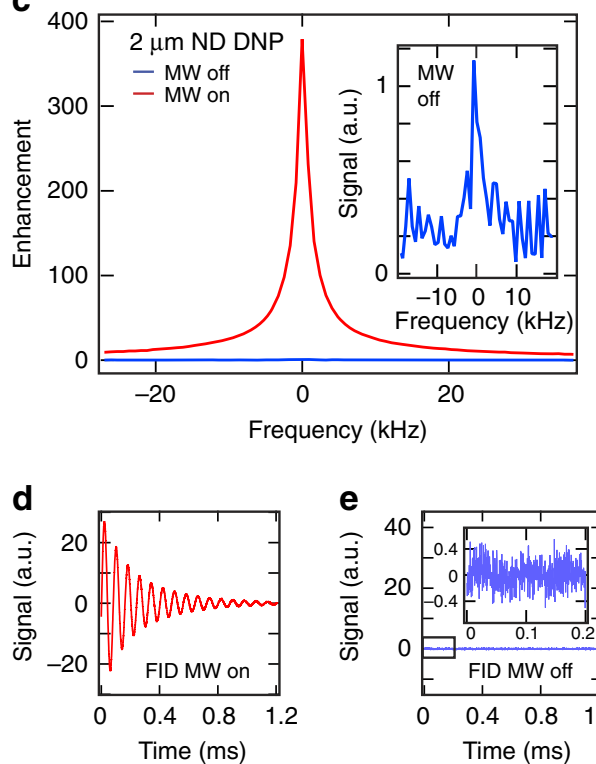

f
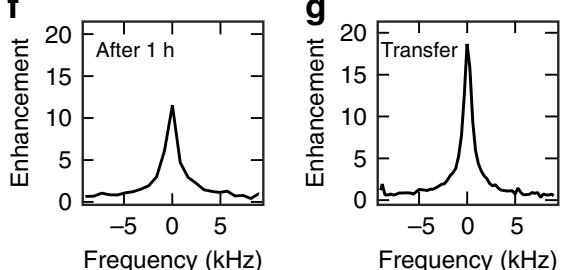

$\mathbf{h}$

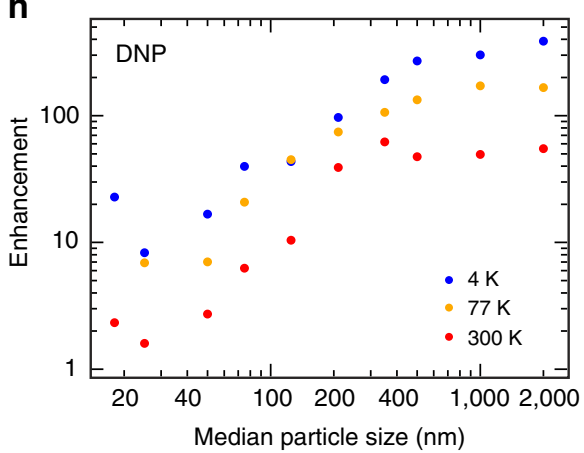

Figure 2 | Dynamic nuclear polarization of ND. (a,b) DNP via the solid effect is used to hyperpolarize ND. (a) Energy level diagram for a dipolar-coupled electron spin-1/2 (e) and a nuclear spin-1/2 (n) system in a magnetic field. The ESR at frequency $\omega_{\mathrm{e}}$ (green), NMR at frequency $\omega_{\mathrm{n}}$ (purple), flip-flop at frequency $\omega_{\mathrm{e}}-\omega_{\mathrm{n}}$ (blue), and flip-flip at frequency $\omega_{\mathrm{e}}+\omega_{\mathrm{n}}$ (red) transitions are shown. Driven flip-flop transitions involve a mutual electron flip and a nuclear flop resulting in a positive nuclear polarization. Driven flip-flip transitions result in a negative nuclear polarization. (b) Schematic showing positive and negative nuclear polarization when driving flip-flop and flip-flip transitions, respectively. (c) Hyperpolarized signal enhancement of 380 times for 2 - $\mu \mathrm{m}$ ND at $T=4 \mathrm{~K}$. Inset: zoom of NMR signal taken at thermal equilibrium ( $T=4 \mathrm{~K})$ with no microwaves. (d,e) NMR free induction decay of $2-\mu \mathrm{m}$ ND after a $\pi / 2$-pulse at $T=4 \mathrm{~K}$. (d) Signal after hyperpolarization. (e) Thermal polarization signal. Inset: zoom of the signal. (f) Signal enhancement of 2- $\mu \mathrm{m}$ ND that was polarized for $1 \mathrm{~h}$ and then allowed to decay at field $(B=3 \mathrm{~T})$ for $1 \mathrm{~h}$. $(\mathbf{g})$ The $2-\mu \mathrm{m}$ ND signal enhancement after transfer from the polarizer to a $B=7 \mathrm{~T}$ magnet for detection. The $2-\mu \mathrm{m}$ ND was polarized for $15 \mathrm{~min}$ at $T=4 \mathrm{~K}$. The transfer took place in a field of $730 \mathrm{mT}$ and took $\sim 15 \mathrm{~s}$. (h) Signal enhancement as a function of particle size and temperature at $T=4 \mathrm{~K}$ (blue), $77 \mathrm{~K}$ (yellow) and $300 \mathrm{~K}$ (red). The enhancement is given by the hyperpolarized signal divided by the thermal signal at each temperature.
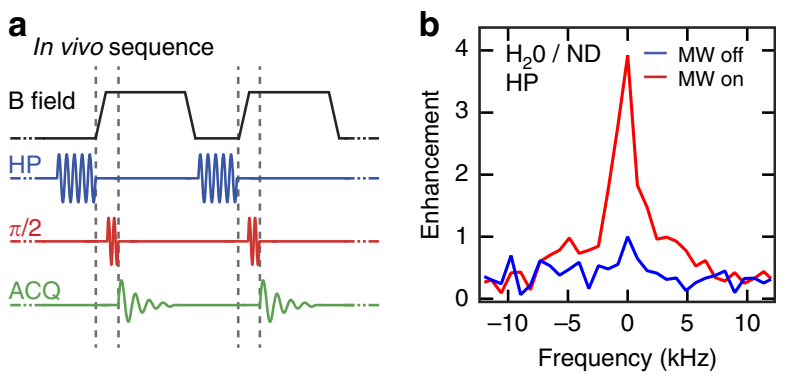

Figure 3 | ND hyperpolarization in the precence of water. (a) The combination of room temperature signal enhancement and long relaxation times opens the possibility of in vivo hyperpolarization using a magnetic field shuttling technique. With the ND agents already administered to the biosystem, hyperpolarization (HP) is performed at low field where microwave heating is reduced. The field is then ramped on a timescale much shorter than $T_{1}$ to enable imaging and detection of the hyperpolarized ND. Schematic shows a repeated sequence with radio frequency $\pi / 2$ -pulses and acquisition window (ACQ). (b) Hyperpolarization in the presence of water, mimicking in vivo conditions. Even at the high microwave frequency of $80 \mathrm{GHz}$, we observe an enhanced signal (red) of approximately four times compared with the thermal polarization (blue).

heating but requires sufficiently long $T_{1}$ times to enable magnetic field ramping between polarization and high-field detection conditions ${ }^{39}$. Hyperpolarized ND appears well suited to explore this modality, since, for example, the signal from 350-nm ND (as a dry powder) can be enhanced by a factor of 40 at room temperature and exhibits a $T_{1}$ of several minutes (long enough for field ramping). Since the polarize and detection sequence can be cycled many times in vivo (Fig. 3a), background noise can be averaged well below the limits imposed by one-shot polarize and detect methods.

To test this modality, we hyperpolarize a slurry of 125-nm ND and water $(200 \mu \mathrm{l}$ water with $\sim 50$-mg ND) at room temperature. Even in the presence of $\sim 80 \mathrm{GHz}$ microwaves, we observe a fourfold enhancement of ${ }^{13} \mathrm{C}$ magnetic resonance signal from the diamond with little discernible heating of the water (Fig. 3b). This enhancement, which corresponds to a halving of the signal relative to the case without water, suggests that such modalities may be possible for small animals.

ND impurity selection and surface modification. The results presented so far are for ND samples readily obtained commercially, without further treatment or surface modification. We now turn to examine the role that surface impurities play in both hyperpolarization and relaxation, noting that there is significant scope to tailor the surface chemistry via passivation and treatment ${ }^{40}$. Our approach is to effectively perform ESR spectroscopy at the magnetic field used for DNP $(B \sim 3 \mathrm{~T})$, by monitoring the NMR signal enhancement as a function of microwave frequency (Fig. 4a; Supplementary Fig. 6). At room temperature, we observe enhancement spectra consisting of four peaks that are in agreement with the low-field ESR data shown in Fig. 1e. The position of these peaks correspond to DNP processes at $\omega_{\mathrm{e}} \pm \omega_{\mathrm{n}}$. As the temperature is lowered, these distinct peaks become dipolar broadened. The high-field DNP spectra are similarly broadened as the particle size is increased, as shown in Fig. 4b.

Modifying the defects or the types of defects within the NDs can change the DNP spectra, leading to enhanced polarization 


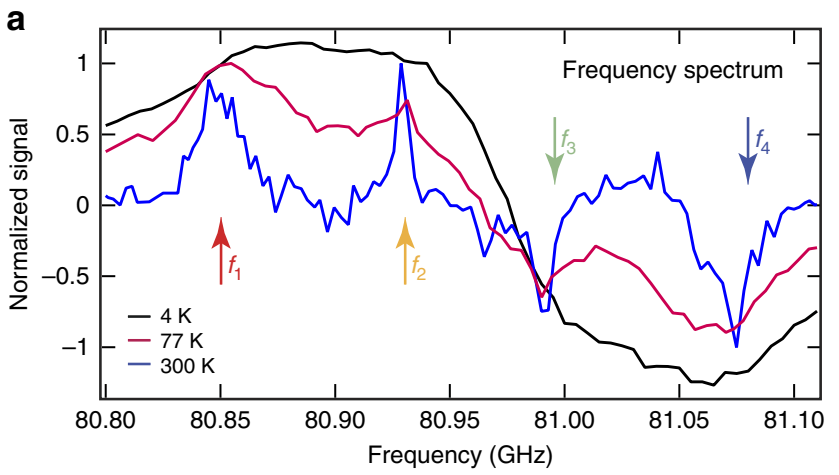

b

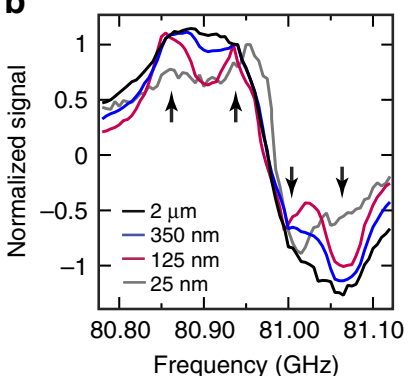

C
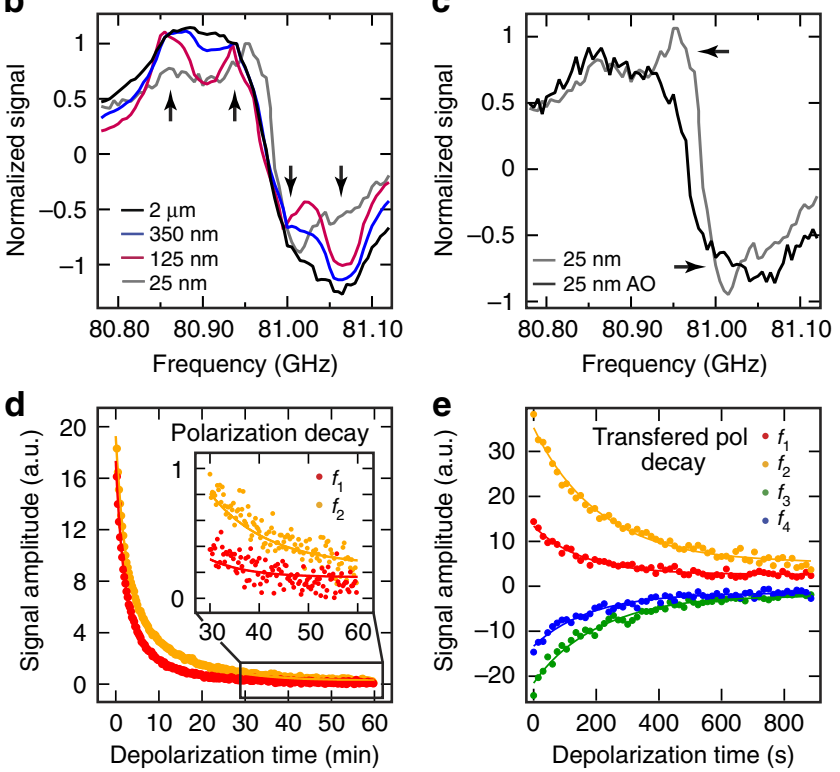

Figure 4 | ND impurity selection and surface modification.

(a) Hyperpolarized signal of $2-\mu \mathrm{m} \mathrm{ND}$ as a function of polarization frequency at $T=4 \mathrm{~K}$ (black), $77 \mathrm{~K}$ (purple) and $300 \mathrm{~K}$ (blue). The arrows indicate the four frequencies at which polarization build-up and decay were examined. The traces were normalized to 1 . Note the features broaden as temperature decreases. (b) Normalized hyperpolarized signal of 2- $\mu \mathrm{m}$ ND (black), 350-nm ND (blue), 125-nm ND (purple) and 25-nm ND (grey) at $T=4 \mathrm{~K}$ as a function of microwave frequency. More features are visible as the particle size decreases. (c) Comparison of the hyperpolarized signal amplitude as a function of frequency for $25-\mathrm{nm}$ ND and $25 \mathrm{~nm}$ air oxidized ND. (d) Depolarization of the hyperpolarized signal of 350-nm ND at $T=4 \mathrm{~K}$ using a small tip angle pulse sequence $\left(\theta=3.5^{\circ}, \mathrm{TR}=15 \mathrm{~s}\right)$ The ND was polarized for $180 \mathrm{~min}$ at the frequencies indicated by the arrows in $\mathbf{a}$. We see a difference in the decay times of the red trace $\left(f_{1}=80.870 \mathrm{GHz}\right.$, $T_{1}=12$ and $\left.2.1 \mathrm{~s}\right)$ and the yellow trace $\left(f_{2}=80.930 \mathrm{GHz}, T_{1}=16\right.$ and $\left.2.5 \mathrm{~s}\right)$. For $\mathbf{d}$ and $\mathbf{e}$, the data has been multiplied by $\cos (\theta)^{n-1}$ to take into account the polarization lost due to the small tip angle. Fits to the data (solid lines) are double exponential decay curves. (e) Depolarization of the signal from 350-nm ND after hyperpolarization for $15 \mathrm{~min}$ at $T=4 \mathrm{~K}$ at the four frequencies indicated by arrows in $\mathbf{a}$ and transfer to a $T=7 \mathrm{~T}$ magnet for detection. The decay was examined using a small tip angle sequence $\left(\theta=8^{\circ}, \mathrm{TR}=15 \mathrm{~s}\right)$. Data are shown in red $\left(f_{1}=80.870 \mathrm{GHz}, T_{1}=157 \mathrm{~s}\right)$, yellow $\left(f_{2}=80.925 \mathrm{GHz}, T_{1}=250 \mathrm{~s}\right)$, green $\left(f_{3}=80.990 \mathrm{GHz}, T_{1}=190 \mathrm{~s}\right)$ and blue $\left(f_{4}=81.050 \mathrm{GHz}, T_{1}=157 \mathrm{~s}\right)$.

and longer relaxation times. We find that burning off the outer layer of ND using air oxidation processes ${ }^{41}$ removes some of the broad spin-1/2 component associated with impurities near the surface of the ND. This can be seen in Fig. $4 c$ where we compare the hyperpolarization spectra of $25 \mathrm{~nm}$ air oxidation ND (black) with standard 25-nm ND (grey). The oxidation process leads to a suppression of the two central lines $\left(f_{2}\right.$ and $\left.f_{3}\right)$ in the spectrum, consistent with removing some of the surface impurities that would otherwise contribute to the signal.

By adjusting the microwave frequency for DNP, we can select different impurity sites for use in hyperpolarization. Polarization via the P1-centres, for instance, can be selected by irradiating at the frequencies corresponding to the outer peaks $\left(f_{1}\right.$ and $\left.f_{4}\right)$ in Fig. 4a. This is in contrast to irradiating at the inner peaks $\left(f_{2}\right.$ and $f_{3}$ ), which also comprise both narrow and broad components from spin-1/2 sites (see discussion of Fig. 1e). Surprisingly, we find that hyperpolarization due to microwave driving at the inner peaks takes longer to build-up, and is retained longer than when driving at the outer peaks associated with the P1-centres. This behaviour is seen in Fig. 4d (Supplementary Figs 7 and 8; Supplementary Tables 1 and 2), where for $350-\mathrm{nm} \mathrm{ND}$, we compare the decay of hyperpolarization established by driving at peak $f_{1}$ or peak $f_{2}$ in Fig. 4 a. These results suggest that nuclear spin diffusion is somewhat suppressed in these systems, since relaxation appears dominated by the particular impurities selected for polarization via the choice of microwave frequency.

Beyond examining the spin dynamics of $\mathrm{ND}$, these results are of practical interest in optimizing conditions for maximum signal enhancement with minimal relaxation. As an example, we compare the signal from $350 \mathrm{~nm} \mathrm{ND}$, initially irradiated with microwaves at the four distinct frequencies indicated in Fig. $4 \mathrm{a}$ and then subsequently transferred to a $B=7 \mathrm{~T}$ system for detection. Following sample transfer, the relaxation data in Fig. $4 \mathrm{e}$ show that a larger polarization is maintained if the nuclei were polarized using the two central spectra peaks $\left(f_{2}\right.$ and $\left.f_{3}\right)$, in comparison with the outer peaks associated with the P1-centres $\left(f_{1}\right.$ and $\left.f_{4}\right)$.

\section{Discussion}

There is significant scope to further enhance the degree of hyperpolarization in ND. In particular, by adding microwave capability to our brute force hyperpolarizer at $\mathrm{mK}$ temperature (based on a dilution refrigerator), much larger polarizations appear possible. Eliminating isolated defects via surface passivation techniques ${ }^{40}$ will also likely result in longer relaxation times. Another direction is to work with isotopically enriched ${ }^{13} \mathrm{C}$ NDs to increase the number of spins that contribute to the signal and enhance spin diffusion from polarization sites on the surface to the spins in the core. Finally, we mention the possibility of using NDs for long-time storage of nuclear polarization that is transferred to the hydrogen nuclei in an aqueous environment using cross-polarization sequences ${ }^{42,43}$. Although the efficiency for polarization transfer is yet to be experimentally explored, this modality may enable life-science applications in which ND is tracked and imaged using standard MRI technology ${ }^{43}$.

\section{Methods}

Nanodiamonds. The NDs used in these experiments were purchased from Microdiamant. The diamonds are synthesized using the high-pressure hightemperature technique. The sizes of the NDs are given as a distribution and a median size. We refer to the diamonds by their median size. Measurements were recorded on MSY 0-0.030, (0-30 nm, median 18), MSY 0-0.05 (0-50 nm, median $25 \mathrm{~nm})$, MSY 0-0.1 (0-100 nm, median $50 \mathrm{~nm})$, MSY 0-0.15 $(0-150 \mathrm{~nm}$, median $75 \mathrm{~nm})$, MSY 0-0.25 (0-250 nm, median $125 \mathrm{~nm})$, MSY 0-500 (0-500 nm, median $210 \mathrm{~nm})$, MSY $0.25-0.5(250-500 \mathrm{~nm}$, median $350 \mathrm{~nm})$, MSY $0.25-0.75$ $(250-750 \mathrm{~nm}$, median $500 \mathrm{~nm})$, MSY 0.75-1.25 (750-1250 nm, median $1000 \mathrm{~nm})$ and MSY 1.5-2.5 (1500-2500 nm, median $2000 \mathrm{~nm})$.

SEM images. SEM measurements were recorded using a Zeiss Ultra Plus Gemini SEM spectrometer working in transmission mode. Suspensions were made of these 
NDs in water and a small amount of the suspension was placed on a TEM grid. The ND size distributions were confirmed (Supplementary Fig. 1).

ESR measurements. ESR measurements were recorded using a Bruker EMX-plus X-Band ESR Spectrometer. The cavity Q ranged between 5,000 for small ND particles and 10,000 for large ND particles. ESR spectra were taken at $0.25 \mu \mathrm{W}$, (within the linear regime of the saturation curves of the impurities) at a modulation amplitude of $1 \mathrm{Gs}$ and a modulation frequency of $100 \mathrm{kHz}$. Each of the three components were simulated separately using Easyspin ${ }^{44}$ and added together to make the final spectrum. Linewidth, signal amplitude and g-factor were varied. The best fit to the data was performed using a least squares analysis.

$\boldsymbol{T}_{\mathbf{1}}$ relaxation. NMR measurements to determine the $T_{1}$ relaxation times at $B=7 \mathrm{~T}$ were made using a saturation recovery pulse sequence that involved $64 \times \pi / 2$ saturation pulses to null any initial polarization followed by a varied time for polarization to build-up, and then a $\pi / 2$ detection pulse. Time domain signals were acquired using a Redstone Tecmag system. Either a stretched exponential (smaller particles) $M=M_{0}\left(1-\exp \left(-\left(t / T_{1}\right)^{\alpha}\right)\right.$ or a double exponential (larger particles) was fitted to the curve (Supplementary Fig. 4). Each ND size was measured three times and the average of the $T_{1}$ times is plotted.

Brute force $\boldsymbol{T}_{\mathbf{1}}$ measurements. The decay of brute force hyperpolarization in Fig. $1 \mathrm{~g}$ (red dots) was measured using a small tip angle detection sequence $\left(\theta=17^{\circ}\right.$, $\mathrm{TR}=15 \mathrm{~s})$. The solid red line is a fit to $M=M_{0} \cos (\theta)^{n-1} e^{-(n-1) \mathrm{TR} / T_{1}}$ resulting in a $T_{1}$ of $53 \mathrm{~min}$. The decay is a combination of the $T_{1}$ decay of the particles and the signal lost due to rf-induced polarization loss with tip angle $\theta$ where $n$ is the $n$th pulse and TR is the repetition time. Every fifth peak is shown in black.

Hyperpolarization measurements. Measurements were recorded at a field of $2.88 \mathrm{~T}$ using a Redstone Tecmag system and a in-house constructed NMR probe (design details can be found in ref 45) inside an Oxford Instruments flow cryostat. The microwave source was a tuneable Gunn Oscillator $(80.5-81.5 \mathrm{GHz})$ combined with a power amplifier. Microwaves were coupled with the sample using a waveguide. Polarization transfer measurements were determined using an in-house constructed NMR spectrometer based on a National Instruments system and an NMR probe at a field of $7 \mathrm{~T}$. Before polarization the signal was saturated with $64 \times \pi / 2$ pulses to null any signal. Enhancement measurements were recorded by hyperpolarizing the $\mathrm{ND}$ at $f=80.855 \mathrm{GHz}(4 \mathrm{~K}, 77 \mathrm{~K})$ and $80.85 \mathrm{GHz}(300 \mathrm{~K})$ and then detecting the signal with a $\pi / 2$-pulse. The hyperpolarized signal was compared with the NMR signal with no microwaves and the same polarization build-up time. Frequency sweeps: the frequency was swept between 80.78 and $81.12 \mathrm{GHz}$ in discrete steps of $5 \mathrm{MHz}$ and polarization was measured at every point. The $2-\mu \mathrm{m} \mathrm{ND}$ was polarized for $30 \mathrm{~s}, 3 \mathrm{~min}$ and $3 \mathrm{~min}$ at $T=4 \mathrm{~K}, 77 \mathrm{~K}$ and $300 \mathrm{~K}$, respectively. The spectra have been normalized to 1 for easier comparison.

Depolarization. Measurements at $\mathrm{B}=7 \mathrm{~T}$ : the ND was polarized for $15 \mathrm{~min}$ at four frequencies ( $f=80.87,80.925,80.99$ and $81.05 \mathrm{GHz}$ in successive experiments) and then transferred to a $B=7 \mathrm{~T}$ magnet for detection. The transfer was performed in a field of $B \sim 0.7 \mathrm{~T}$, created from rare-earth permanent magnets and took $\sim 20 \mathrm{~s}$. A small tip angle pulse sequence with $8^{\circ}$ pulses was used to detect the signal. Enhancement is compared with ND at thermal equilibrium at $B=7 \mathrm{~T}$. Measurements at $\mathrm{B}=2.88 \mathrm{~T}$ : the ND was polarized for $180 \mathrm{~min}$ at two frequencies and the decay was monitored with small tip angle pulses (every $15 \mathrm{~s}$ ). Depolarization data were multiplied by $\cos (\alpha)^{(n-1)}$ to take into account rf-induced depolarization. The resulting data were fitted with a double exponential.

\section{References}

1. Wang, M. \& Thanou, M. Targeting nanoparticles to cancer. Pharmacol. Res. 62, 90-99 (2010).

2. Li, C. A targeted approach to cancer imaging and therapy. Nat. Mater. 13, 110-115 (2014).

3. Getts, D. R. et al. Therapeutic inflammatory monocyte modulation using immune-modifying microparticles. Sci. Transl. Med. 6, 219ra7 (2014).

4. Gupta, A. S. Nanomedicine approaches in vascular disease: a review. Nanomedicine 7, 763-779 (2011).

5. Mura, S. \& Couvreur, P. Nanotheranostics for personalized medicine. $A d v$. Drug Deliv. Rev. 64, 1394-1416 (2012).

6. Cassidy, M. C., Chan, H. R., Ross, B. D., Bhattacharya, P. K. \& Marcus, C. M. In vivo magnetic resonance imaging of hyperpolarized silicon particles. Nat. Nanotechnol. 8, 363-368 (2013).

7. Cassidy, M. C., Ramanathan, C., Cory, D. G., Ager, J. W. \& Marcus, C. M. Radical-free dynamic nuclear polarization using electronic defects in silicon. Phys. Rev. B 87, 161306 (2013).

8. Liu, K.-K., Cheng, C.-L., Chang, C.-C. \& Chao, J.-I. Biocompatible and detectable carboxylated nanodiamond on human cell. Nanotechnology 18, 325102 (2007)
9. Huang, H., Pierstorff, E., Osawa, E. \& Ho, D. Active nanodiamond hydrogels for chemotherapeutic delivery. Nano Lett. 7, 3305-3314 (2007).

10. Kruger, A., Liang, Y., Jarre, G. \& Stegk, J. Surface functionalisation of detonation diamond suitable for biological applications. J. Mater. Chem. 16, 2322-2328 (2006).

11. Say, J. M. et al. Luminescent nanodiamonds for biomedical applications. Biophys. Rev. 3, 171-184 (2011).

12. Chow, E. K. et al. Nanodiamond therapeutic delivery agents mediate enhanced chemoresistant tumor treatment. Sci. Transl. Med. 3, 73ra21 (2011).

13. Chen, M. et al. Nanodiamond-mediated delivery of water-insoluble therapeutics. ACS Nano 3, 2016-2022 (2009).

14. Zhang, X.-Q. et al. Polymer-functionalized nanodiamond platforms as vehicles for gene delivery. ACS Nano 3, 2609-2616 (2009).

15. McGuinness, L. P. et al. Quantum measurement and orientation tracking of fluorescent nanodiamonds inside living cells. Nat. Nanotechnol. 6, 358-363 (2011).

16. Maze, J. R. et al. Nanoscale magnetic sensing with an individual electronic spin in diamond. Nature 455, 644-647 (2008).

17. Balasubramanian, G. et al. Nanoscale imaging magnetometry with diamond spins under ambient conditions. Nature 455, 648-651 (2008).

18. Bhallamudi, V. P. \& Hammel, P. C. Nitrogen-vacancy centres: Nanoscale MRI Nature Nanotechnol. 10, 104-106 (2015).

19. Rugar, D. et al. Proton magnetic resonance imaging using a nitrogen-vacancy spin sensor. Nature Nanotechnol. 10, 120-124 (2015).

20. Häberle, T., Schmid-Lorch, D., Reinhard, F. \& Wrachtrup, J. Nanoscale nuclear magnetic imaging with chemical contrast. Nature Nanotechnol. 10, 125-128 (2015).

21. DeVience, S. J. et al. Nanoscale NMR spectroscopy and imaging of multiple nuclear species. Nature Nanotechnol. 10, 129-134 (2015).

22. Brenneis, A. et al. Ultrafast electronic readout of diamond nitrogen-vacancy centres coupled to graphene. Nature Nanotechnol. 10, 135-139 (2015).

23. Keshari, K. R. \& Wilson, D. M. Chemistry and biochemistry of ${ }^{13} \mathrm{C}$ hyperpolarized magnetic resonance using dynamic nuclear polarization. Chem Soc. Rev. 43, 1627-1659 (2014).

24. Ardenkjaer-Larsen, J. H. et al. Increase in signal-to-noise ratio of $>10,000$ times in liquid-state NMR. Proc. Natl Acad. Sci. USA 100, 10158-10163 (2003).

25. Day, S. E. et al. Detecting tumor response to treatment using hyperpolarized ${ }^{13} \mathrm{C}$ magnetic resonance imaging and spectroscopy. Nat. Med 13, 1382-1387 (2007).

26. Nelson, S. J. et al. Metabolic imaging of patients with prostate cancer using hyperpolarized $\left[1{ }^{13}\right.$ C]pyruvate. Sci. Transl. Med. 5, 198 ra108 (2013).

27. Hoch, M. J. R. \& Reynhardt, E. C. Nuclear spin-lattice relaxation of dilute spins in semiconducting diamond. Phys. Rev. B 37, 9222-9226 (1998).

28. King, J. P., Coles, P. J. \& Reimer, J. A. Optical polarization of ${ }^{13} \mathrm{C}$ nuclei in diamond through nitrogen vacancy centers. Phys. Rev. B 81, 073201 (2010).

29. Fischer, R., Jarmola, A., Kehayias, P. \& Budker, D. Optical polarization of nuclear ensembles in diamond. Phys. Rev. B 87, 125207 (2013).

30. Alvarez, G. A. et al.Local and bulk ${ }^{13} \mathrm{C}$ hyperpolarization in NV-centered diamonds at variable fields and orientations. Preprint at http://arxiv.org/abs/ 1412.8635 (2014).

31. King, J. P. et al.Room-temperature in situ nuclear spin hyperpolarization from optically-pumped nitrogen vacancy centers in diamond. Preprint at http:// arxiv.org/abs/1501.02897 (2015).

32. Panich, A. M. et al. Nuclear magnetic resonance study of ultrananocrystalline diamonds. Eur. Phys. J. B 52, 397-402 (2006).

33. Shames, A. I. et al. Defects and impurities in nanodiamonds: EPR, NMR and TEM study. J. Phys. Chem. Solids 63, 1993-2001 (2002).

34. Casabianca, L. B., Shames, A. I., Panich, A. M., Shenderova, O. \& Frydman, L. Factors affecting DNP NMR in polycrystalline diamond samples. J. Phys. Chem. C 115, 19041-19048 (2011).

35. Dutta, P., Martinez, G. V. \& Gillies, R. J. Nanodiamond as a new hyperpolarizing agent and its ${ }^{13} \mathrm{C}$ MRS. J. Phys. Chem. Lett. 5, 597-600 (2014)

36. Furman, G. B., Kunoff, E. M., Goren, S. D., Pasquier, V. \& Tinet, D. Nuclear spin-lattice relaxation via paramagnetic impurities in solids with arbitrary space dimension. Phys. Rev. B 52, 10182-10187 (1995).

37. Blumberg, W. E. Nuclear spin-lattice relaxation caused by paramagnetic impurities. Phys. Rev. 119, 79-84 (1960).

38. Abragam, A. \& Goldman, M. Principles of dynamic nuclear polarisation. Rep. Prog. Phys. 41, 395-467 (1978).

39. Lurie, D. J., Davies, G. R., Foster, M. A. \& Hutchison, J. M. Field-cycled PEDRI imaging of free radicals with detection at $450 \mathrm{mT}$. Magn. Reson. Imaging 23, 175-181 (2005).

40. Baumann, P. K. \& Nemanich, R. J. Surface cleaning, electronic states and electron affinity of diamond (100), (111) and (110) surfaces. Surf. Sci. 409, 320-335 (1998).

41. Gaebel, T. et al. Size-reduction of nanodiamonds via air oxidation. Diam. Relat. Mater. 21, 28-32 (2012).

42. Bornet, A., Melzi, R., Jannin, S. \& Bodenhausen, G. Cross polarization for dissolution dynamic nuclear polarization experiments at readily 
accessible temperatures $1.2<\mathrm{T}<4.2 \mathrm{~K}$. Appl. Magn. Reson. 43, 107-117 (2012).

43. Marco-Rius, I. et al. Quantitation of a spin polarization-induced nuclear Overhauser effect (SPINOE) between a hyperpolarized ${ }^{13} \mathrm{C}$-labeled cell metabolite and water protons. Contrast Media Mol. Imaging 9, 182-186 (2014).

44. Stoll, S. \& Schweiger, A. Easyspin, a comprehensive software package for spectral simulation and analysis in EPR. J. Magn. Reson. 178, 42-55 (2006).

45. Cassidy, M. C. Hyperpolarized silicon particles as in-vivo imaging agents. Ph.D. thesisHarvard Univ. (2012).

\section{Acknowledgements}

We thank M. Cassidy for technical contributions in the construction of the hyperpolarization probe and M. Cassidy and C. Marcus for useful discussions. For SEM measurements, the authors acknowledge the facilities and technical assistance of the Australian Centre for Microscopy \& Microanalysis at the University of Sydney. For ESR measurements, the authors acknowledge the staff and facilities at the Nuclear Magnetic Resonance Facility at the Mark Wainwright Analytical Centre at the University of New South Wales. This work is supported by the Australian Research Council Centre of Excellence Scheme (Grant No. EQuS CE110001013) ARC DP1094439, and the Lockheed Martin Corporation.

\section{Author contributions}

E.R., T.G. and T.B. performed the experiment. All authors interpreted, processed and analysed the data. All authors contributed to writing the manuscript.

\section{Additional information}

Supplementary Information accompanies this paper at http://www.nature.com/ naturecommunications

Competing financial interests: The authors declare no competing financial interests.

Reprints and permission information is available online at http://npg.nature.com/ reprintsandpermissions/

How to cite this article: Rej, E. et al. Hyperpolarized nanodiamond with long spin-relaxation times. Nat. Commun. 6:8459 doi: 10.1038/ncomms9459 (2015)

(c) (i) This work is licensed under a Creative Commons Attribution 4.0 International License. The images or other third party material in this article are included in the article's Creative Commons license, unless indicated otherwise in the credit line; if the material is not included under the Creative Commons license, users will need to obtain permission from the license holder to reproduce the material. To view a copy of this license, visit http://creativecommons.org/licenses/by/4.0/ 